\section{ralud mental}

Ulloa Flores, Rosa Elena; Contreras Hernández, Cecilia; Paniagua Navarrete, Karina; Victoria Figueroa, Gamaliel

Frecuencia de autolesiones y características clínicas asociadas en adolescentes que acudieron a un hospital psiquiátrico infantil

Salud Mental, vol. 36, núm. 5, septiembre-octubre, 2013, pp. 417-420

Instituto Nacional de Psiquiatría Ramón de la Fuente Muñiz

Distrito Federal, México
Salud Mental

perezrh@imp.edu.mx

Instituto Nacional de Psiquiatría Ramón

de la Fuente Muñiz

México

Disponible en: http://www.redalyc.org/articulo.oa?id=58228970010

- Cómo citar el artículo

- Número completo

- Más información del artículo

- Página de la revista en redalyc.org 


\title{
Frecuencia de autolesiones y características clínicas asociadas en adolescentes que acudieron a un hospital psiquiátrico infantil
}

\author{
Rosa Elena Ulloa Flores, ${ }^{1}$ Cecilia Contreras Hernández, ${ }^{2}$ Karina Paniagua Navarrete, ${ }^{3}$ \\ Gamaliel Victoria Figueroa'
}

Artículo original

\section{SUMMARY}

Introduction

Although studies of self-harm in adolescents have pointed to psychopathology as a risk factor, the information on the phenomenon in clinical population of Mexican adolescents is scarce.

\section{Methods}

This study examined demographic and clinical characteristics, as well as types, frequency and reasons for self-harm in 556 adolescents attending a child psychiatric hospital from 2005 to 2011 through record review.

\section{Results}

High frequencies of female gender, low socioeconomic status, family violence and sexual abuse history were found. The most common diagnoses were affective disorders, conduct disorders and substance abuse. Self-cutting was the most used method and the main reasons for self-harm included low frustration tolerance, attention seek, and symptoms of anxiety and mood disorders. An increase in cases was observed over time, particularly in patients with mood disorders and/ or substance abuse.

\section{Conclusions}

Self-harm in adolescents with psychopathology has increased and is frequently associated with depressive and conduct disorders. It is important to determine the presence of self-harm in the assessment of these patients.

Key words: Self-harm, adolescents, psychopathology, prevalence, self-cutting, Mexican.

\section{RESUMEN}

\section{Introducción}

Aunque los estudios de autolesiones en adolescentes han señalado a la psicopatología como un factor de riesgo, existe poca información del fenómeno en población clínica en México.

\section{Método}

El presente estudio examinó las características demográficas y clínicas, así como los tipos, frecuencia y motivos para autolesionarse de 556 adolescentes que acudieron a un hospital psiquiátrico infantil de 2005 a 2011 por medio de la revisión de su expediente.

\section{Resultados}

Dentro de las características que se encontraron con mayor frecuencia fueron el sexo femenino, el nivel socioeconómico bajo, la violencia intrafamiliar y el antecedente de abuso sexual. Los diagnósticos más frecuentes fueron los trastornos afectivos, los trastornos de conducta y el abuso de sustancias. El corte fue el método más empleado y los principales motivos para autolesionarse incluían la baja tolerancia a la frustración, el llamar la atención de otros, los síntomas afectivos y ansiosos. Se observó un incremento de casos a lo largo del tiempo, en particular aquellos asociados a trastornos afectivos y abuso de sustancias.

\section{Conclusiones}

Las autolesiones en adolescentes con psicopatología se han incrementado y están frecuentemente asociadas a trastornos depresivos y de conducta. Es importante determinar la presencia de autolesiones durante la evaluación inicial de estos pacientes.

Palabras clave: Autolesiones, adolescentes, psicopatología, prevalencia, cortes, mexicanos.

\section{INTRODUCCION}

Las autolesiones se definen como actos intencionales y autodirigidos que ocasionan daños corporales de baja letalidad reali- zados para disminuir el estrés. ${ }^{1}$ En estudios conducidos en países como Australia se ha reportado que el 8.1\% de la población general se ha autolesionado en algún momento de la vida. ${ }^{2}$ Específicamente, algunos estudios de revisión muestran que

Departamento de Psicofalmacología del desarrollo. Hospital Psiquiátrico Infantil "Dr. Juan N Navarro", México.

Centro Michoacano de Salud Mental, Tarímbaro, Michoacán, México.

Hospital General de Zona Número 6, IMSS Ciudad Juárez, Chihuahua, México.

Correspondencia: Dra. Rosa Elena Ulloa. Hospital Psiquiátrico Infantil "Dr. Juan N Navarro". San Buenaventura 86, Col. Belisario Domínguez, Tlalpan,

14080, México, DF. E-mail: eulloa@hotmail.com

Recibido primera versión: 11 de julio de 2012. Segunda versión: 9 de mayo de 2013. Aceptado: 7 de junio de 2013. 
en adolescentes las prevalencias van del $13 \mathrm{al} 23.2 \%{ }^{3}$ y en la población que acude a los hospitales por atención se ha reportado un incremento de este fenómeno a lo largo del tiempo. ${ }^{4}$

Las formas más frecuentes de autolesión corresponden a cortes, golpes y quemaduras; menos frecuentes son la inserción de objetos bajo la piel, el rascarse en exceso, el morderse, el arrancarse el cabello o la ingesta de cuerpos o líquidos extraños. ${ }^{5}$

Diversas son las razones por las cuales lo hacen los sujetos que se autolesionan, como la búsqueda de regulación afectiva, el control de impulsos ${ }^{6-8}$ o llamar la atención de otros. ${ }^{9}$ Numerosos factores se han asociado a esta conducta en los adolescentes, entre los que se cuentan la presencia de psicopatología en ellos o sus familiares, la disfunción familiar, la presencia de adversidad social ${ }^{10}$ o la ocurrencia de eventos estresantes. ${ }^{11}$ La relación entre psicopatología y conducta autolesiva ha sido mostrada en estudios de adultos, donde se reporta que el $90 \%$ de los sujetos con autolesiones tiene al menos un trastorno psiquiátrico. ${ }^{12}$ Uno de los estudios más importantes de autolesiones en adolescentes es el estudio basado en población escolar CASE (Child and Adolescent Self-harm in Europe), en el que cerca de 30000 adolescentes de 15 y 16 años completaron un cuestionario anónimo sobre autolesiones, un reporte de eventos estresantes y escalas para evaluar depresión, ansiedad e impulsividad. Los resultados de este estudio muestran que las autolesiones estaban relacionadas con síntomas de depresión, ansiedad e impulsividad, además de eventos estresantes. ${ }^{13}$

Los resultados de estudios en adolescentes de muestras clínicas en varios países han replicado este hallazgo, mostrando además una asociación con los trastornos externalizados. ${ }^{14-16}$

Hasta la fecha no existen reportes de la frecuencia de autolesiones en población clínica de adolescentes mexicanos. Los estudios en Europa y en Estados Unidos de América han mostrado que los individuos con dicha conducta constituyen un grupo heterogéneo, por lo que se requiere información acerca de la frecuencia y características asociadas a las autolesiones en nuestra población.

El presente estudio se diseñó con el objetivo de describir las características demográficas, clínicas y de funcionamiento familiar en adolescentes con autolesiones y psicopatología atendidos en el Hospital Psiquiátrico Infantil "Dr. Juan N Navarro" (HPIJNN), así como determinar si se han registrado cambios en la frecuencia, en el tipo de autolesiones o en la comorbilidad psiquiátrica en esta población a lo largo del tiempo.

\section{MÉTODO}

El presente estudio consistió en una revisión de todos los expedientes de adolescentes de 12 a 17 años, atendidos por autolesiones en el periodo comprendido entre los años 2005 y 2011 en el Hospital Psiquiátrico Infantil "Dr. Juan N. Navarro", en la Ciudad de México.
Durante dicha revisión, para identificar los casos de interés se examinó la historia clínica, la descripción del padecimiento y la nota de ingreso al servicio que el paciente requería. Una vez localizados los casos, se obtuvieron los siguientes datos: a) conducta autolesiva: frecuencia, tipo, motivos para realizarla, tiempo de evolución; b) datos demográficos y clínicos: edad, sexo, religión, nivel socioeconómico, motivo de consulta y diagnóstico; c) composición y funcionamiento familiar, y d) antecedente de abuso sexual.

Los datos se analizaron con el programa estadístico PASW Statistics 18 utilizando estadística descriptiva.

\section{RESULTADOS}

La muestra se integró por los expedientes clínicos de los 556 pacientes que presentaban autolesiones y correspondieron al 9.8\% de los adolescentes atendidos durante el periodo señalado. Sus características demográficas y diagnósticos se muestran en el cuadro 1. Los trastornos afectivos (trastorno depresivo mayor, trastorno distímico, trastorno bipolar y trastorno adaptativo con ánimo depresivo) fueron los más comúnmente asignados a los pacientes, seguidos por los trastornos de conducta (que incluían trastorno por déficit de

Cuadro 1. Características demográficas y clínicas de la muestra

\begin{tabular}{lcr}
\hline Característica & $\%$ & $\mathrm{~N}=556$ \\
\hline Sexo femenino & 76.30 & 424 \\
Ocupación: & 77.20 & 429 \\
- Estudiantes & 3.40 & 18 \\
- Empleados & 19.20 & 106 \\
- Ninguna & & \\
Nivel socioeconómico bajo & 71.20 & 396 \\
Religión católica & 80.00 & 445 \\
Constitución familiar: & & \\
- Ambos padres & 47.30 & 263 \\
- Sólo la madre & 38.70 & 215 \\
- Otros familiares/casa hogar & 14.00 & 78 \\
Disfunción/violencia familiar & 83.50 & 464 \\
& 49.50 & 275 \\
Abuso sexual & 29.70 & 165 \\
Edad promedio & $14.46 \pm 1.50$ años \\
Escolaridad promedio & $7.70 \pm 1.65$ años \\
Diagnósticos & & \\
- Trastorno afectivo & 67.10 & 373 \\
- Trastorno de conducta & 23.00 & 128 \\
- Abuso de sustancias & 21.80 & 121 \\
- Trastorno de ansiedad & 13.10 & 73 \\
- Personalidad & 9.20 & 51 \\
- Trastorno de alimentación & 6.70 & 37 \\
- Retraso mental & 5.80 & 32 \\
- Esquizofrenia & 3.10 & 17 \\
- Epilepsia & 2.50 & 14 \\
- Trastorno de eliminación & 1.80 & 10 \\
- Tricotilomanía & 1.30 & 7 \\
\hline
\end{tabular}


atención con hiperactividad, trastorno negativista desafiante, trastorno disocial) y el abuso de sustancias.

Los pacientes estudiados refirieron diversos motivos de consulta. Los más frecuentes fueron los problemas de conducta $(27 \%)$, la conducta suicida $(23.9 \%)$ y la presencia de autolesiones $(22.5 \%)$. Otros motivos de consulta fueron síntomas depresivos (12.4\%), síntomas de ansiedad (7.7\%) y el consumo de sustancias (5.2\%). El 43.3\% acudió por iniciativa de sus padres, $41.7 \%$ fueron referidos por otras instituciones de salud, por las escuelas $(9.2 \%)$ o por la Procuraduría General de Justicia (5.9\%).

En la figura 1 se observan los diferentes tipos de autolesiones autoinflingidas, los cortes superficiales en muñecas y antebrazos, los golpes y los rasguños fueron las más frecuentes.

En cuanto a los motivos para autolesionarse, en $42.1 \%$ de los casos se asociaba a baja tolerancia a la frustración y manipulación; en los expedientes se mencionaba que las lesiones eran posteriores a discutir con los padres o las parejas sentimentales, de forma impulsiva, con el objeto de disminuir la frustración y/o para provocar culpa en la persona con la que discutían. En el 30.6\% de los casos esta conducta se asoció a síntomas afectivos como tristeza o irritabilidad o para "lograr sentir algo y sentirse vivos". En el 19.1\% estuvo asociada a síntomas de ansiedad; en el $4.5 \%$, a síntomas psicóticos, generalmente alucinaciones auditivas imperativas; en el 1.6\% de los casos las autolesiones fueron conductas imitativas y en el $1.4 \%$ se realizaron para pertenecer a un grupo. $\mathrm{Al}$ momento de su evaluación clínica, se reportó que la conducta autolesiva había estado presente durante $18.48( \pm 16.8)$ meses, siendo frecuente (cuatro o más veces por mes) en $43 \%$ de los casos.

Se observó un incremento notable de casos de adolescentes con autolesiones atendidos en el hospital: en 2005 esta conducta se registró en $0.4 \%$ de la población atendida y para el 2011 se había incrementado a 5.7\%, en particular la frecuencia de cortes y golpes en los últimos tres años. Se observaron cambios en la frecuencia de los diagnósticos asociados a las autolesiones: los trastornos afectivos y el abuso de sustancias

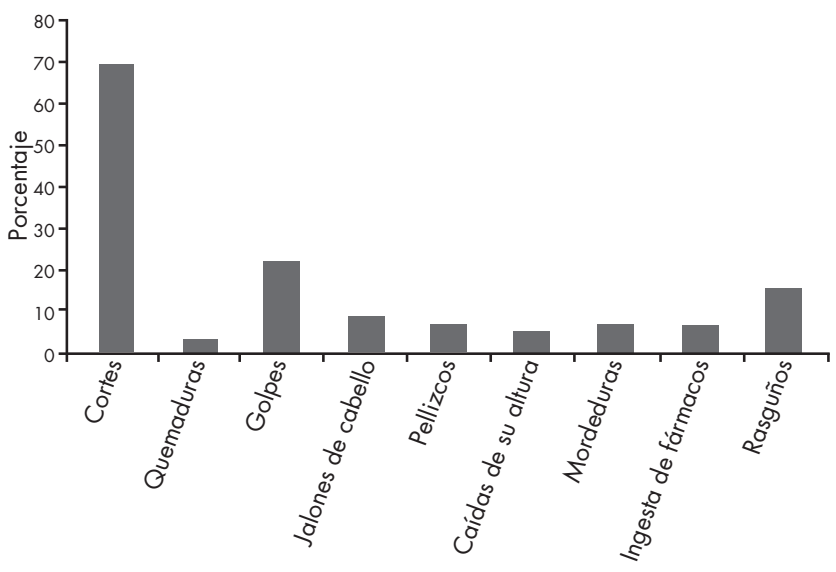

Figura 1. Frecuencia de los diferentes tipos de autolesiones

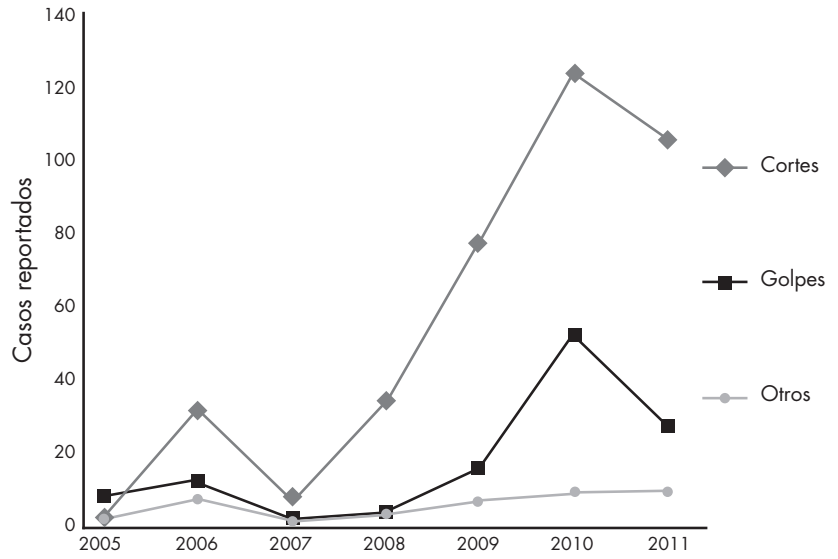

Figura 2. Frecuencia de autolesiones por año

se incrementaron del 50 al $73 \%$ y del 0 al 18\%, respectivamente; por otro lado, las autolesiones asociadas a retraso mental se redujeron del 14\% en 2005 al 3\% en 2011 (figura 2).

\section{DISCUSIÓN}

La incidencia de autolesiones y las características demográficas de la población clínica estudiada fueron similares a las reportadas en estudios previos. ${ }^{17,18} \mathrm{El}$ nivel socioeconómico bajo observado en la mayor parte de estos pacientes se ha descrito como un factor de riesgo. ${ }^{19}$ Asimismo, la alta frecuencia de alteraciones en el funcionamiento familiar y el antecedente de abuso sexual presentes en esta muestra han sido reportados como factores de mayor peso. ${ }^{10,20-22}$ Se sabe que las malas prácticas parentales y el maltrato pueden incrementar el riesgo de autolesiones, ya que están asociadas a las dificultades interpersonales durante la adolescencia, frecuentemente descritas en los sujetos con autolesiones. ${ }^{23}$

Los resultados mostraron que los pacientes se habían autolesionado por periodos prolongados antes de solicitar ayuda especializada, lo cual puede explicarse por la poca advertencia que tienen los padres y maestros sobre la frecuencia de este fenómeno y su impacto en la salud de los adolescentes. ${ }^{24}$

La alta frecuencia de trastornos afectivos y por consumo de sustancias en esta muestra semeja a las de estudios en otros países. Es importante hacer notar que 23\% de los casos tenían trastornos de conducta. Aunque estos diagnósticos no se han señalado como factores de riesgo, la impulsividad, uno de sus síntomas cardinales, es frecuentemente reportada en pacientes con autolesiones. ${ }^{19}$ Diversos autores han sugerido la necesidad de explorar a profundidad el papel de los trastornos externalizados y el consumo de sustancias en las autolesiones, cuya asociación con la conducta suicida ha sido reportada. ${ }^{25}$

La literatura médica señala que las formas más frecuentes de autolesiones en adolescentes son cortes, golpes, quemaduras, mordeduras, rasguños, pellizcos y jalarse el cabe$110,{ }^{5}$ lo cual concuerda con el presente estudio, en particular 
con la alta frecuencia de cortes ${ }^{17}$ Los problemas de conducta y los síntomas afectivos fueron las causas principalmente asociadas a las autolesiones en los adolescentes de este estudio, en semejanza al reporte de Shin. ${ }^{26} \mathrm{El}$ incremento de autolesiones en pacientes con abuso de sustancias muestra la necesidad de evaluar esta conducta en todos los pacientes adolescentes, no sólo en aquellos con trastornos afectivos.

Es muy importante hacer notar que el reporte de autolesiones en la población del HPIJNN se incrementó 14 veces durante los seis años estudiados, lo cual supera el incremento reportado por O'Loughlin. ${ }^{27}$ Esto podría explicarse por la inadvertencia del fenómeno en años anteriores, o reflejar un incremento real del fenómeno en adolescentes con psicopatología.

Los resultados de este estudio deben examinarse tomando en cuenta las limitaciones de un estudio basado en la revisión de expedientes, donde no se registra de manera uniforme la gravedad de los síntomas ni de la disfunción asociada a la patología de los pacientes.

\section{CONCLUSIONES}

La prevalencia de autolesiones en adolescentes mexicanos con psicopatología se ha incrementado notoriamente a lo largo del tiempo; estas lesiones están asociadas principalmente a trastornos afectivos y de conducta. La distribución por sexo y el tipo de autolesiones son similares a las reportadas en otros países. Es importante incluir dentro de la valoración psiquiátrica cotidiana la búsqueda intencional de conductas autolesivas en pacientes de cualquier edad, en este caso adolescentes, que acudan por cualquier motivo, en especial por problemas de conducta, síntomas afectivos o ansiosos, o bien por consumo de sustancias y que además tengan una importante carga de adversidad psicosocial o de eventos estresantes de vida.

\section{AGRADECIMIENTOS}

Los autores agradecen la colaboración de Aldair Cabrera y Gabriela López en la realización de este manuscrito.

\section{REFERENCIAS}

1. Walsh B. Treating self-injury: A practical guide. New York: Tercera edición; Guilford Press; 2005.

2. Taylor A, Martin G, Dal Grande E, Swannell S et al. Methodological issues associated with collecting sensitive information over the telephone-experience from an Australian non-suicidal self-injury (NSSI) prevalence study. BMC Med Res Methodol 2011;11:20.http://www. ncbi.nlm.nih.gov/pmc/articles/PMC3049141/?tool=pubmed. Consultado el 8 de mayo de 2012.

3. Jacobson C, Gould M. The epidemiology and phenomenology of nonsuicidal self-injurious behavior among adolescents: a critical review of the literature. Arch Suicide Res 2007;11:129-147.

4. Wilkinson S, Taylor G, Templeton L, Mistral W et al. Admissions to hospital for deliberate self-harm in England 1995-2000: an analysis of hospital episode statistics. J Public Health Med 2002;24:179-183.

5. MacAniff L, Kiselica M. Understanding and counseling self-mutilation in female adolescents and young adults. J Counseling Development 2001;79:46-52.
6. Claes L, Jiménez-Murcia S, Agüera Z, Castro R et al. Male eating disorder patients with and without non-suicidal self-injury: A comparison of psychopathological and personality features. Eur Eat Disord Rev 2011. http://dx.doi.org/10.1002/erv.1161. Consultado el 24 de febrero de 2012.

7. Groholt B, Ekeberg O, Haldorsen T. Adolescents hospitalised with deliberate self-harm: the significance of an intention to die. Eur Child Adolesc Psychiatry 2000;9:244-254.

8. Rodham K, Hawton K, Evans E. Reasons for deliberate self-harm: comparison of self-poisoners and self-cutters in a community sample of adolescents. J Am Acad Child Adolesc Psychiatry 2004;43:80-87.

9. Scoliers G, Portzky G, Madge N, Hewitt A et al. Reasons for adolescent deliberate self-harm: a cry of pain and/or a cry for help? Findings from the child and adolescent self-harm in Europe (CASE) study. Soc Psychiatry Psychiatr Epidemiol 2009;44:601-607.

10. Ayton A, Rasool H, Cottrell D. Deliberate self-harm in children and adolescents: association with social deprivation. Eur Child Adolesc Psychiatry 2003;12:303-307.

11. Shawn MH, Ali B, Bossarte RM, Van Dulmen M et al. Self-harm and suicide attempts among high-risk, urban youth in the US: Shared and unique risk and protective factors. Int J Environ Res Public Health 2012;9:178-191.

12. Haw C, Hawton K, Houston K, Townsend E. Psychiatric and personality disorders in deliberate self-harm patients. Br J Psychiatry 2001;178:48-54.

13. Madge N, Hawton K, McMahon E, Corcoran P et al. Psychological characteristics, stressful life events and deliberate self-harm: findings from the Child \& Adolescent Self-harm in Europe (CASE) Study. Eur Child Adolesc Psychiatry 2011;10:499-508.

14. Kirkcaldy B, Brown J, Siefen R. Disruptive behavioural disorders, self harm and suicidal ideation among German adolescents in psychiatric care. Int J Adolesc Med Health 2006;18:597-614.

15. Csorba J, Dinya E, Plener P, Nagy E et al. Clinical diagnoses, characteristics of risk behaviour, differences between suicidal and non-suicidal subgroups of Hungarian adolescent outpatients practising selfinjury. Eur Child Adolesc Psychiatry 2009;18:309-320.

16. Cerutti R, Manca M, Presaghi F, Gratz K. Prevalence and clinical correlates of deliberate self-harm among a community sample of Italian adolescents. J Adolesc 2011;34:337-347.

17. Morey C, Corcoran P, Arensman E, Perry I. The prevalence of self-reported deliberate self harm in Irish adolescents. BMC Public Health 2008;8:79. doi: 10.1186/1471-2458-8-79. Consultado el 2 de junio de 2012.

18. Nichols P. Bad body fever and deliberate self-injury. Reclaiming Children Youth 2000;9:151-156.

19. Skegg K. Self-harm. Lancet 2005;366:1471-1483.

20. Boxer P. Variations in risk and treatment factors among adolescents engaging in different types of deliberate self-harm in an inpatient sample. J Clin Child Adolesc Psychol 2010;39:470-480.

21. Romans S, Martin J, Anderson J, Herbison G et al. Sexual abuse in childhood and deliberate self-harm. Am J Psychiatry 1995;152:1336-1342.

22. Gladstone G, Parker G, Mitchell P, Malhi G et al. Implications of childhood trauma for depressed women: an analysis of pathways from childhood sexual abuse to deliberate self-harm and revictimization. Am J Psychiatry 2004;161:1417-1425.

23. Johnson J, Cohen P, Gould M, Kasen $S$ et al. Childhood adversities, interpersonal difficulties, and risk for suicide attempts during late adolescence and early adulthood. Arch Gen Psychiatry 2002;59:741-749.

24. Portzky G, De Wilde E, van Heeringen K. Deliberate self-harm in young people: differences in prevalence and risk factors between the Netherlands and Belgium. Eur Child Adolesc Psychiatry 2008;17:179-186.

25. Verona E, Sachs-Ericsson N, Joiner TJ. Suicide attempts associated with externalizing psychopathology in an epidemiological sample. Am J Psychiatry 2004;161:444-451.

26. Shin Y, Chung Y, Lim K, Lee $Y$ et al. Childhood predictors of deliberate self-harm behavior and suicide ideation in Korean adolescents: a prospective population-based follow-up study. J Korean Med Sci 2009;24:215-222.

27. O'Loughlin S, Sherwood J. A 20-year review of trends in deliberate self-harm in a British town, 1981-2000. Soc Psychiatry Psychiatr Epidemiol 2005;40:446-453.

Artículo sin conflicto de intereses 